\title{
A 28-Year-Old Woman with Ascites and Multiple Focal Spleen Lesions
}

\author{
Alice Pirovano ${ }^{1}$, Erica Matino $^{1}$, Erika Zecca $^{1}$, Martina Costanzo $^{1}$, Alessandro Croce $^{1}$, \\ Monica Leutner ${ }^{2}$, Raffaele Romito ${ }^{2}$, Mario Pirisi ${ }^{1,2}$ \\ ${ }^{1}$ Università del Piemonte Orientale, Novara, Italy \\ ${ }^{2}$ Azienda Ospedaliero-Universitaria Maggiore della Carità, Novara, Italy
}

Received: 30/01/2019

Accepted: $15 / 04 / 2019$

Published: 06/05/2019

How to cite this article: Pirovano A, Matino E, Zecca E, Costanzo M, Croce A, Leutner M, Romito R, Pirisi M. A 28-year-old woman with ascites and multiple focal spleen lesions. EJCRIM 2019;6: doi:10.12890/2019_001061.

Conflicts of Interests: The Authors declare that there are no competing interests.

Acknowledgements: The authors are grateful to Professor Ettore Bartoli for useful suggestions and careful reading of the manuscript.

This article is licensed under a Commons Attribution Non-Commercial 4.0 License

\section{HOSPITAL GRAND ROUNDS}

Public University Hospital Maggiore della Carità, Novara, Italy by Editorial Board Member, Prof. Ettore Bartoli

The Public University Hospital "Maggiore della Carità", Novara, Italy, is a 700-bed teaching hospital, with 2700 employees. Approximately 500 of these are medical doctors. It is the home Institution for the Medical School of the "Università del Piemonte Orientale". In its early days it was a branch of the University of Turin and it became autonomous in 1998. Today, it admits 150 medical students and 80 interns a year and is strongly committed to pursuing excellence in education and research. In fact, according to the most recent CENSIS survey, it ranks $6^{\text {th }}$ out of the 37 state universities nationwide.

Despite its recent history as a university hospital, the roots of the "Maggiore" date back to the Middle Ages. Originally it was a charity institute dedicated to the Archangel Michael - hence the name "Casa di San Michele della Carità".It was founded in the XI century with the aim of helping the poor, the elderly and the pilgrims. The care activity was addressed to aiding the sick only later, starting from the end of the thirteenth century. In 1482, a Bull of Pope Sixtus IV imposed the union between the Hospital of "S. Michele" with the other seven hospitals in Novara, confirming the importance assumed by this institution in its urban context.

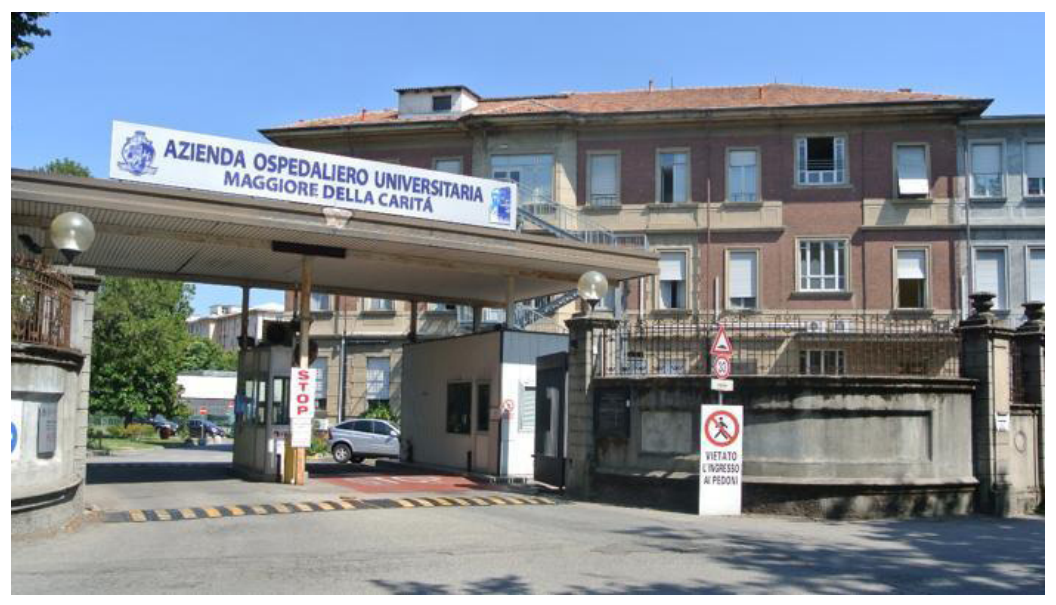




\section{ABSTRACT}

Serous effusions complicating the course of lymphomas occur commonly in the pleural space but seldom in the peritoneum, where they most often present as chylous ascites with diagnostic cytology. Almost invariably, in these rare cases, the serum to ascites albumin gradient is low. We describe a 28-year-old woman with anasarca, ascites and a serum to ascites albumin gradient of $1.1 \mathrm{~g} / \mathrm{dl}$, consistent with portal hypertension. No tumour cells were detected in the ascitic fluid. However, a CT scan of the chest and abdomen disclosed liver and spleen enlargement and multiple enlarged retroperitoneal lymph nodes, suspicious for a lymphoproliferative disorder. Bone marrow aspiration and biopsy were not diagnostic, so a decision was made to proceed with a splenectomy despite the onset of low-grade disseminated intravascular coagulation.

Surgery was uneventful. Diffuse large B cell lymphoma was diagnosed. A liver biopsy taken at the time of surgery demonstrated that the liver parenchyma was massively infiltrated by reactive T lymphocytes surrounding rare large CD20+ tumour cells. This infiltrate had likely led to increased portal pressure attended by ascites formation, which resolved completely after chemotherapy. The case emphasizes the rewards of pursuing a diagnosis supported by a high prior probability even in the presence of apparently discordant laboratory findings, as well as the importance of performing a diagnostic splenectomy in case of splenomegaly with unexplained focal lesions.

\section{LEARNING POINTS}

- Lymphomas may present with serous effusion, which is usually chylous and with positive cytology when represented by ascites accumulation; non-chylous effusions can be due to altered lymphatic drainage, extrinsic compression of the portal vein by enlarged lymph nodes as well as massive infiltration of the liver by lymphoma.

- If the cause of splenomegaly is unclear, diagnostic splenectomy remains a viable option.

- The diagnosis of lymphoma should always be pursued, even if it requires apparently unwise surgery, since this type of cancer can be treated effectively only if thoroughly characterized pathologically and molecularly.

\section{KEYWORDS}

Ascites, splenomegaly, lymphoma, disseminated intravascular coagulation, diagnostic splenectomy

\section{CASE DESCRIPTION}

A 28-year-old woman was admitted to the internal medicine division of an academic hospital in northern Italy with complaints of progressive abdominal distention and fatigue. She also described substantial weight gain and anorexia over the previous 6 months.

At physical examination, the patient appeared chronically ill. She was afebrile and all other vital parameters were normal, but there was anasarca, with marked swelling and erythema of the lower legs. There were no enlarged or palpable lymph nodes, but a protuberant abdomen, with central tympany and flank dullness at percussion, was noted. The remainder of the examination was normal. A full blood count revealed white blood cells (WBC) $7.69 \times 10^{9} /$ ( $81 \%$ neutrophils, $9 \%$ lymphocytes), red blood cells (RBC) $3.96 \times 10^{12} /$, haemoglobin $11.2 \mathrm{~g} / \mathrm{l}$, and platelets (PLTs) $143 \times 10^{9} / \mathrm{l}$.

Examination of a peripheral blood smear did not add significant information. Prothrombin time was 14.4 sec. Serological studies for human immunodeficiency virus, hepatitis B virus and hepatitis C virus were negative. Other laboratory results included lactate dehydrogenase (LDH) $688 \mathrm{IU} / \mathrm{I}$ (normal limits, 208-450 IU/I), total serum proteins $3.8 \mathrm{~g} / \mathrm{dl}$ (6.4-8.3 g/dl), albumin $2.2 \mathrm{~g} / \mathrm{dl}$ (3.5-5.5 g/dl) and total bilirubin 1.5 $\mathrm{mg} / \mathrm{dl}$ (0.3-1.2 mg/dl). Renal function tests and electrolyte levels were normal. A paracentesis was performed with aspiration of a yellowcoloured, slightly turbid fluid whose analysis gave the following results: albumin $1.1 \mathrm{~g} / \mathrm{dl}$ (serum to ascites albumin gradient of $1.1 \mathrm{~g} / \mathrm{dl}$ ), WBC

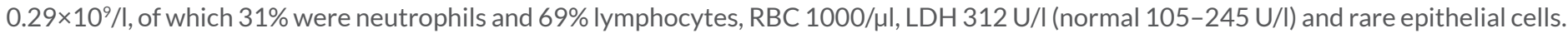
Culture of the ascitic fluid and real time-polymerase chain reaction amplification for Mycobacterium tuberculosis were negative.

A computed tomography (CT) scan of the abdomen and chest confirmed massive ascites, liver enlargement without focal lesions, thinning of the inferior vena cava and hepatic veins, enlargement of the spleen (longitudinal diameter, $15 \mathrm{~cm}$ ) whose parenchymal architecture was distorted by several lesions (the largest being $5.38 \mathrm{~cm}$ in diameter), multiple enlarged retroperitoneal lymph nodes above the psoas muscle, small bilateral pleural effusions, and diffuse imbibition of the adipose tissue (Figs. 1 and 2).

A pig-tail catheter was left in the abdomen for symptomatic relief. A positron emission tomography (PET) scan confirmed the presence of abdominal polyadenopathy, with iliac, para-aortic, retroperitoneal and para-caval localization, and the involvement of liver and spleen due to a suspected neoplastic condition. A bone marrow aspiration and a biopsy were performed: cellularity was normal for age, as were the myeloid and erythroid series, with a slight maturation defect of myeloid cells; mild non-specific infiltration by $\mathrm{T}$ lymphocytes was also present. 


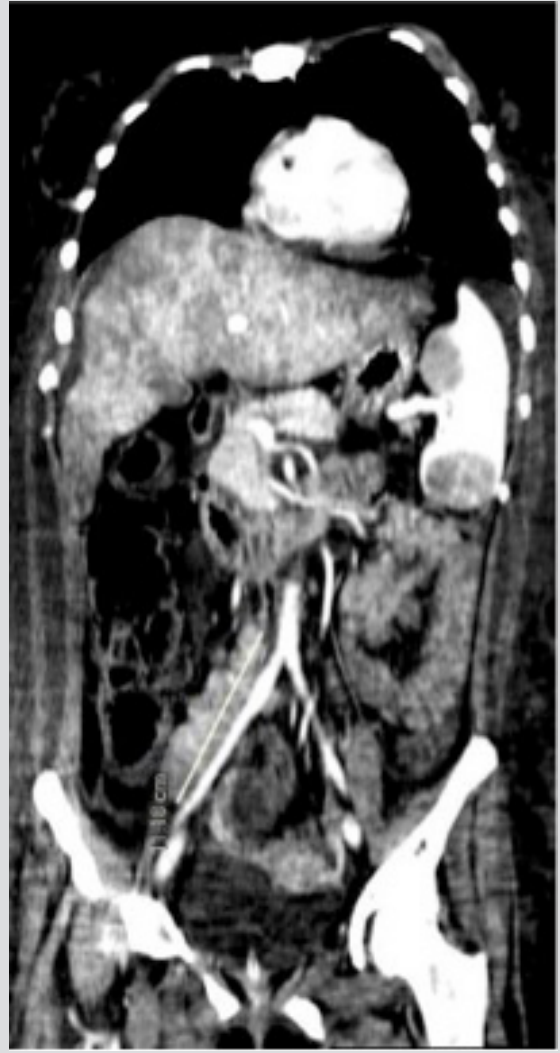

Figure 1. Coronal view of a contrast-enhanced CT scan of the abdomen and chest, showing a distorted and enlarged spleen and a diffusely enlarged liver with no focal lesions

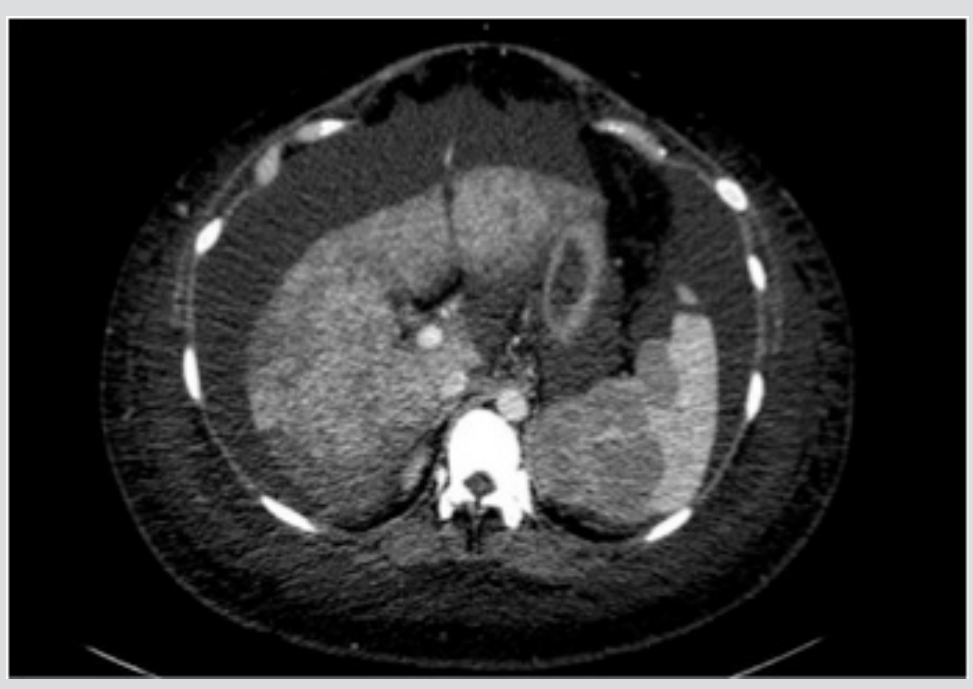

Figure 2. Transverse view of a contrast-enhanced CT scan of the abdomen, showing a distorted and enlarged spleen and a diffusely enlarged liver with no focal lesions, with recanalization of the umbilical vein

Despite stable self-reported conditions, the blood count and coagulation studies became consistent with low-grade disseminated intravascular coagulation (DIC) (haemoglobin $8.3 \mathrm{~g} / \mathrm{dl}$, PLTs 44×10\%/I, INR 1.59, activated partial thromboplastin time $39.9 \mathrm{sec}$ ). Based on a working diagnosis of a lymphoproliferative disease, intravenous corticosteroids and fresh frozen plasma infusions were started and the decision was taken to proceed to diagnostic splenectomy and liver biopsy during laparotomy (Figs. 3-5).

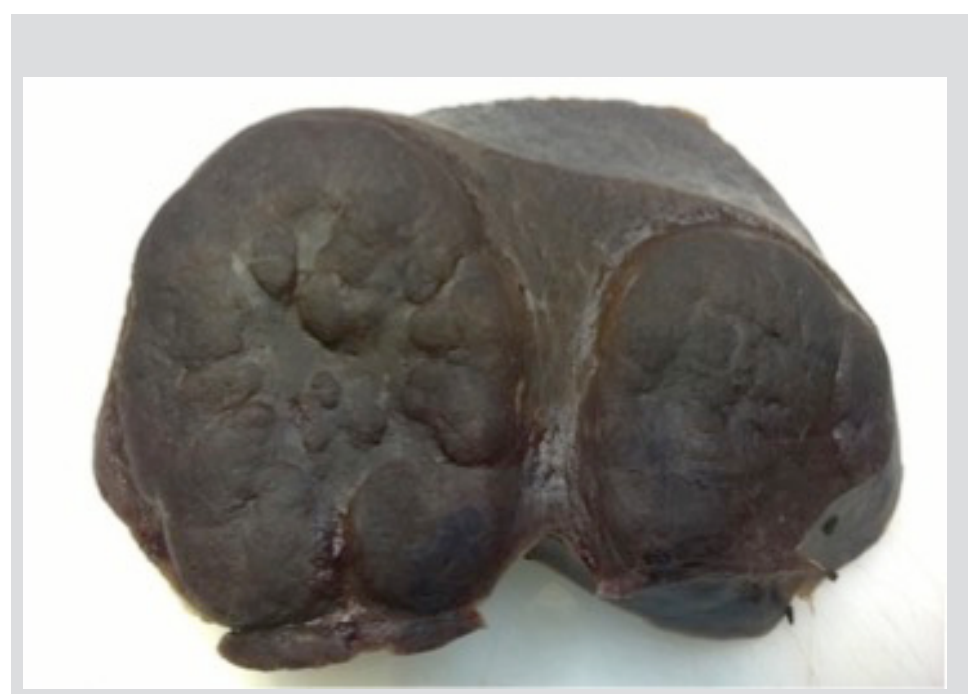

Figure 3. Gross examination of the spleen showing marked splenomegaly and multiple large nodules, with pushing borders, evenly dark red coloured as the surrounding parenchyma

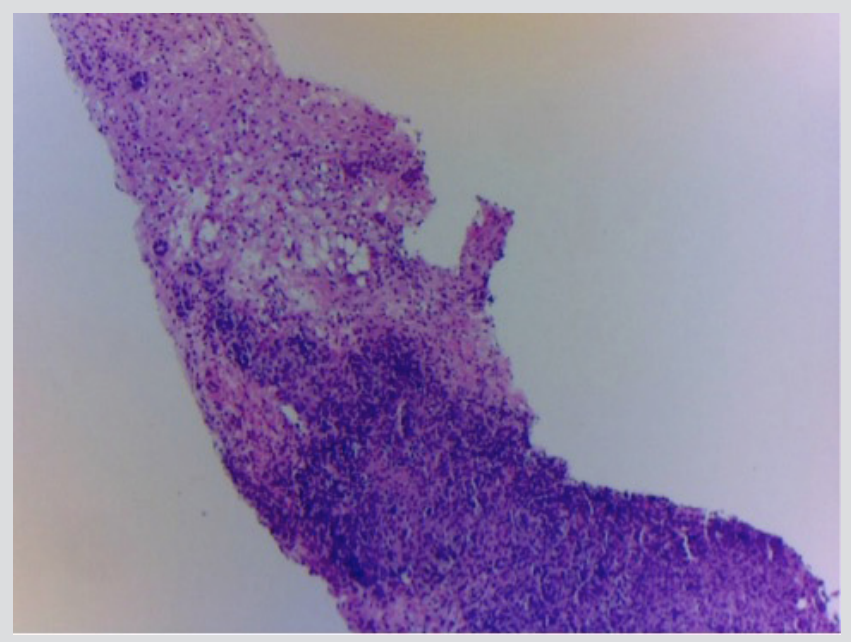

Figure 4. Histopathological examination of the liver biopsy specimen taken at the time of laparotomy showing nodular infiltration of the liver parenchyma by mononuclear cells (H\&E stain, 10x) 


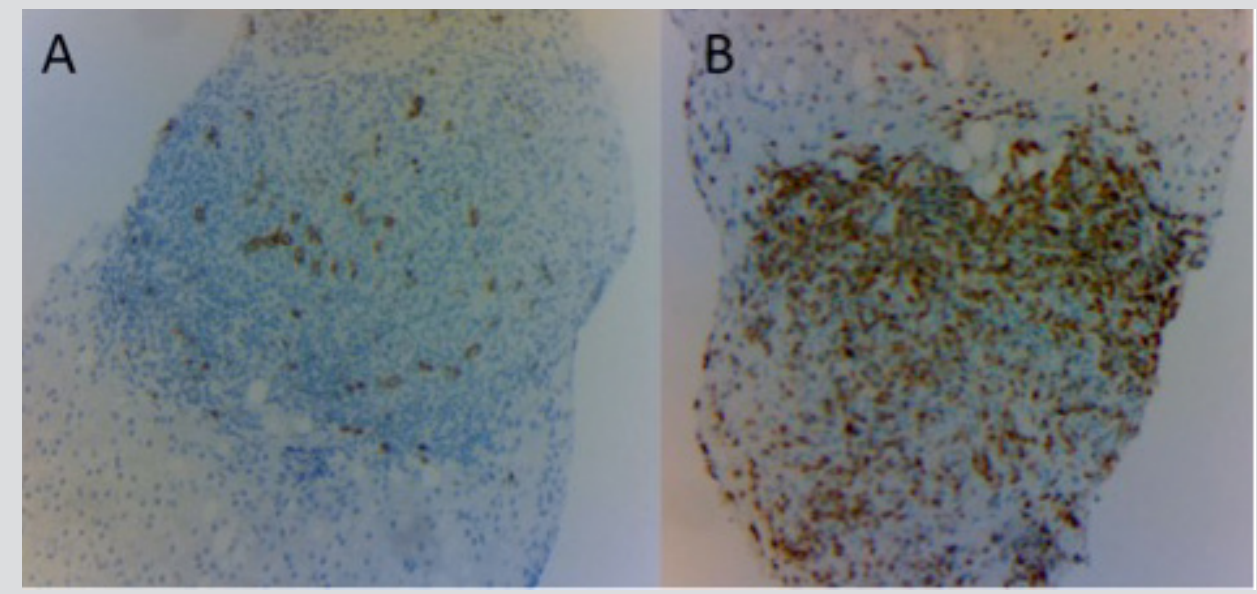

Figure 5. Liver biopsy immunohistochemistry staining showing scattered large $B C D 20+$ lymphocytes (A) and predominantly small T CD3+ lymphocytes (B) (magnification: 10x)

\section{DISCUSSION}

The present case was challenging mainly for two reasons: on the one hand, presentation with ascites characterized by a high serum to ascites albumin gradient, partially inconsistent with the strong leading diagnosis of lymphoma; on the other hand, the need to substantiate this diagnosis with tissue sample(s) enabling complete characterization and classification, as the cytological specimens yielded by ascites examination were inadequate. We will start by discussing the diagnostic approach to ascites.

\section{Diagnostic approach to ascites}

The traditional approach to investigate the cause of ascites requires paracentesis and determination of the serum to ascites albumin gradient. This gradient, calculated by subtracting the albumin concentration in the ascitic fluid from that found concomitantly in serum, accurately identifies the presence of portal hypertension ${ }^{[1]}$. Although by far the most common cause of ascites due to portal hypertension is cirrhosis, a high gradient $(\geq 1.1 \mathrm{~g} / \mathrm{l})$ is by no means specific for cirrhosis, being the result of any condition causing portal hypertension, such as heart failure or Budd-Chiari syndrome. On the other hand, the most common cause of ascites with a low gradient is cancer, especially when it causes peritoneal carcinomatosis. Massive liver infiltration by metastases, neoplastic thrombosis of the hepatic veins, and obstruction of the thoracic ducts are further important causes of malignant ascites.

Occasionally, other tests may be needed to establish the cause of ascites, such as examination of lactate dehydrogenase, amylase and triglyceride concentrations and tests to document infection by M. tuberculosis (including culture, polymerase chain reaction amplification with specific primers, and adenosine deaminase concentration in the ascitic fluid) ${ }^{[1,2]}$. Finally, idiopathic portal hypertension, once called Banti's disease, is characterized by mild to moderate ascites with rapid response to diuretic treatment, hypochromic anaemia, massive splenomegaly and bleeding from oesophageal varices. It is usually diagnosed by performing a liver biopsy and liver haemodynamic studies and excluding cirrhosis and other rarer causes (e.g., congenital hepatic fibrosis) ${ }^{[3]}$.

\section{Ascites occurrence in lymphoma}

Serous effusions often complicate the course of non-Hodgkin and Hodgkin lymphomas. The most common effusion site is the pleural space (20-30\%), followed-far less commonly-by the pericardium and the peritoneum ${ }^{[4]}$. In the rare instances in which lymphomas present with ascites, the mechanisms include impaired lymphatic drainage due to obstruction by enlarged lymph nodes, venous obstruction, infection or radiation therapy ${ }^{[4]}$. Chylous effusions, on the other hand, are always caused by an obstruction of the lymphatic trunks ${ }^{[5]}$. In the present case, the serum to ascites albumin gradient was consistent with the presence of portal hypertension. In retrospect, this finding can be explained by the combination of altered lymphatic drainage, extrinsic compression of the portal vein by enlarged lymph nodes and, in our opinion most importantly, massive infiltration of the liver by lymphoma.

\section{Diagnostic approach to focal spleen lesions}

The most common causes of splenomegaly are liver and haematological diseases, which together explain $60 \%$ of cases ${ }^{[6]}$. Imaging studies are paramount for reaching a diagnosis, particularly in the presence of focal spleen lesions. At contrast-enhanced ultrasound examination, hypo-enhancement in the parenchymal phase is predictive of malignancy in $87 \%$ of cases $^{[7]}$. A CT scan is able to distinguish between 
neoplastic (either benign or malignant) and non-neoplastic diseases (such as an abscess or a cyst) causing spleen enlargement, and it should be considered also in cases in which a cause can be inferred with high pretest probability, for example cirrhosis ${ }^{[8]}$. A PET scan does not offer significant additional information compared to $\mathrm{CT}^{[9]}$. A simplified differential diagnosis of the focal spleen lesions in the present case should include pyogenic abscesses, granulomata, metastasis and lymphoproliferative diseases.

Pyogenic abscesses are most commonly caused by haematogenous spread of infection. On CT they appear as low-attenuation lesions with a central focus of higher attenuation and possibly ring enhancement ${ }^{[8]}$. However, the patient described here did not exhibit clinical manifestations (such as fever, abdominal pain or abdominal tenderness) or laboratory abnormalities related to the presence of abscesses (leucocytosis, elevated bilirubin and/or liver enzymes); furthermore, all blood cultures were negative. Moreover, she did not have any recognized risk factors for splenic abscess formation, such as diabetes mellitus or underlying pancreatic or hepatobiliary disease, including liver transplantation ${ }^{[10]}$.

Granulomatous infection by M. tuberculosis usually occurs in a miliary form caused by haematogenous dissemination. The more common sites in the abdomen include the lymph nodes, genitourinary tract, peritoneal cavity and gastrointestinal tract ${ }^{[10]}$. Hepatic and splenic tuberculosis (TB) can present as organ enlargement, macronodular disease, or with a miliary pattern ${ }^{[11]}$. TB is a major cause of ascites not due to cirrhosis, and usually (but not always) has a low serum to ascites albumin gradient ${ }^{[1]}$. The patient presented here had no history of prior TB infection or disease, known or possible TB exposure, and/or a history of travel to an area where TB is endemic, such as sub-Saharan Africa, India and Southeast Asia ${ }^{[12]}$. The absence of signs and symptoms of infection and a negative chest CT scan argued against TB as the explanation for this case; finally, blood and ascitic fluid cultures and testing of the ascitic fluid for M. tuberculosis were negative.

The most common primary cancers with splenic metastases originate from the skin, breast, lung, ovary, stomach and prostate ${ }^{[13]}$. On $\mathrm{CT}^{\text {, }}$ splenic metastases are typically hypodense, but their appearance can vary depending on the primary tumour ${ }^{[8]}$. Despite the negative cytological findings on the ascitic fluid and the lack of evidence for a primary tumour in other sites, the possibility of a metastatic tumour could not be ruled out in the present case.

On the other hand, lymphoma is the most common malignant tumour of the spleen. Splenic involvement is seen at presentation in $33 \%$ of all patients with Hodgkin lymphoma and in 30-40\% of patients with non-Hodgkin lymphoma. Lymphoma can infiltrate the spleen diffusely, causing splenomegaly, or can present as nodules or masse ${ }^{[14]}$. At CT, lesions are hypodense, but they are more recognizable on PET, since they show marked fludeoxyglucose uptake ${ }^{[8]}$.

One possible approach to determine the diagnosis in this case could have been to perform a percutaneous image-guided fine needle or core biopsy of the spleen. Once deemed a very dangerous procedure, today a splenic biopsy has acceptable complication rates, as shown in the more recent series and metanalysis ${ }^{[15,16]}$. However, we found no documentation in the literature that a splenic biopsy can be done safely in the presence of ascites and/or of DIC, two good reasons, in our opinion, to consider its performance unwise. Ultimately, as lymphoma was one of our differential diagnoses and thus required an excisional biopsy for complete histological, immunophenotypic and genetic studies, the decision to perform a diagnostic splenectomy was taken.

\section{Current indications for diagnostic splenectomy}

The first recorded splenectomy was carried out in 1549, when Adriano Zaccarelli removed the spleen of a young woman in Naples with malaria, who survived at least 6 years after the operation. A better documented splenectomy was attempted almost three centuries later by Quittenbaum, who operated on a woman with portal hypertension and splenomegaly, who unfortunately died perioperatively of exsanguination: a first clue that most of the operative risk of splenectomy is related to the indication rather than to the operation per se. Indeed, the significant hazards associated with surgery, which can have acute (e.g., haemorrhage, acute pancreatitis), short-term (e.g., overwhelming perioperative infection, with/without DIC) and long-term complications (e.g., venous and arterial thrombosis) ${ }^{[17]}$, have mitigated the enthusiasm of many surgeons. By far, trauma remains the primary indication for splenectomy. In the past, surgical removal of the spleen was commonly performed for symptomatic relief in patients with massive splenomegaly and hypersplenism. It was also highly valued to accurately stage Hodgkin and non-Hodgkin lymphomas ${ }^{[18,19]}$, before being replaced by PET-CT. Nowadays, as shown by analysis of a US database (from which splenectomies performed for traumatic injury, vascular anomalies or as part of a pancreatectomy had been excluded), the most common disease-related indication for a splenectomy was immune thrombocytopenia ${ }^{[20]}$. According to this study, out of 31,446 splenectomies (mostly performed at laparotomy), 2,218 (7.1\%) were due to splenic lymphoma, which ranked fifth on the list; diagnostic evaluation in the presence of a splenomegaly was fourth, being the indication for splenectomy in a further 2,708 patients (8.6\%). Of course, in comparison to the 1980s and the 1990s, today we have far more sophisticated tools to reach a diagnosis and to stage a disease. On the other hand, the excellent results of current treatment of lymphoma cannot be obtained without complete pathological and molecular characterization, which usually requires excisional biopsy of a lymph node or of an extra-lymphatic organ. In the present case, a further element to consider in performing a diagnostic splenectomy was the coexistence of DIC. 
DIC worsens the clinical outcome of lymphomas and is associated with an elevated, though not prohibitive, surgical risk ${ }^{[21]}$; on the other hand, the cornerstone of cancer-associated DIC is the treatment of the underlying disorder. In the present case, the benefits of performing a splenectomy clearly outweighed the risks.

\section{DIAGNOSIS AND OUTCOME}

\section{Pathology findings}

The operative and post-operative courses were uneventful. At a gross examination, our patient's spleen weighed $786 \mathrm{~g}$ and measured $15 \times 12$ $\mathrm{cm}$; there were multiple enlarged polycyclic and coalescent nodules, the largest being $8.5 \mathrm{~cm}$ in diameter (Fig. 3). Histological examination of splenic and liver tissue confirmed the diagnosis of diffuse large B cell lymphoma (DLBLC), T cell/histiocyte-rich type.

\section{Treatment and follow-up}

For large B cell lymphomas, the treatment of choice is a combination regimen of cyclophosphamide, hydroxydaunomycin, vincristine and prednisone (CHOP). Since rituximab is a chimeric anti-CD20 IgG1 monoclonal antibody, a cell surface protein that occurs almost exclusively in mature B cells, the combination of rituximab and CHOP (R-CHOP) has become the new standard for patients with DLBLC ${ }^{[22]}$. Our patient was discharged home after completion of her first R-CHOP cycle. She no longer had signs of fluid retention and the pig-tail peritoneal catheter had been removed. Her condition was reportedly excellent at the end of her scheduled treatment regimen.

This case emphasizes the rewards of pursuing a diagnosis predicted with a high prior probability although countered by apparently discordant laboratory findings, even when this requires invasive procedures, such as a diagnostic splenectomy in case of splenomegaly with unexplained focal lesions, highly suspicious for lymphoma.

\section{REFERENCES}

1. Runyon BA. Management of adult patients with ascites due to cirrhosis. Hepatology 2004;39:841-856.

2. Riquelme A, Calvo M, Salech F, Valderrama S, Pattillo A, Arellano M, et al. Value of adenosine deaminase (ADA) in ascitic fluid for the diagnosis of tuberculous peritonitis: a meta-analysis. J Clin Gastroenterol 2006;40:705-710.

3. Schouten JN, Garcia-Pagan JC, Valla DC, Janssen HL. Idiopathic noncirrhotic portal hypertension. Hepatology 2011;54:1071-1081.

4. Das DK. Serous effusions in malignant lymphomas: a review. Diagn Cytopathol 2006;34:335-347.

5. Almakdisi T, Massoud S, Makdisi G. Lymphomas and chylous ascites: review of the literature. Oncologist 2005;10:632-635.

6. O'Reilly RA. Splenomegaly in 2,505 patients at a large university medical center from 1913 to 1995. 1963 to 1995: 449 patients. West J Med 1998;169:88-97.

7. Caremani M, Occhini U, Caremani A, Tacconi D, Lapini L, Accorsi A, et al. Focal splenic lesions: US findings. J Ultrasound 2013;16(2):65-74.

8. Thipphavong S, Duigenan S, Schindera ST, Gee MS, Philips S. Nonneoplastic, benign, and malignant splenic diseases: cross-sectional imaging findings and rare disease entities. AJR Am J Roentgenol 2014;203:315-322.

9. Pugalenthi A, Bradley C, Gonen M, Do KG, Strong V, Jarnagin W, et al. Splenectomy to treat splenic lesions: an analysis of 148 cases at a cancer center. J Surg Oncol 2013;108:521525.

10. Huang CJ, Pitt HA, Lipsett PA, Osterman FA Jr, Lillemoe KD, Cameron JL, et al. Pyogenic hepatic abscess. Changing trends over 42 years. Ann Surg $1996 ; 223: 600-607$.

11. Lee WK, Van Tonder F, Tartaglia CJ, Dagia C, Cazzato RL, Duddalwar VA, et al. CT appearances of abdominal tuberculosis. Clin Radiol 2012;67:596-604.

12. World Health Organization. Global tuberculosis report 2018. Available from https://www.who.int/tb/publications/global_report/en/ (accessed 17 April 2019).

13. Compérat E, Bardier-Dupas A, Camparo P, Capron F, Charlotte F. Splenic metastases: clinicopathologic presentation, differential diagnosis, and pathogenesis. Arch Pathol Lab Med 2007;131:965-969.

14. Saboo SS, Krajewski KM, O'Regan KN, Giardino A, Brown JR, Ramaiya N, et al. Spleen in haematological malignancies: spectrum of imaging findings. Br J Radiol 2012;85:81-92.

15. Olson MC, Atwell TD, Harmsen WS, Konrad A, King RL, Lin Y, et al. Safety and accuracy of percutaneous image-guided core biopsy of the spleen. AJR Am J Roentgenol 2016;206:655-659.

16. Mclnnes MD, Kielar AZ, Macdonald DB. Percutaneous image-guided biopsy of the spleen: systematic review and meta-analysis of the complication rate and diagnostic accuracy. Radiology 2011;260:699-708.

17. Weledij EP. Benefits and risks of splenectomy. Int J Surg 2014;12:113-119.

18. Glatstein E, Guernsey JM, Rosenberg SA, Kaplan H. The value of laparotomy and splenectomy in the staging of Hodgkin's disease. Cancer 1969;24:709-718.

19. Veronesi U, Musumeci R, Pizzetti F, Gennari L, Bonadonna G. Proceedings: The value of staging laparotomy in non-Hodgkin's lymphomas (with emphasis on the histiocytic type). Cancer 1974;33:446-459.

20. Matharoo GS, Afthinos JN, Gibbs KE. Trends in splenectomy: where does laparoscopy stand? JSLS 2014;18(4). pii: e2014.00239.

21. Chi S, Ikezoe T. Disseminated intravascular coagulation in non-Hodgkin lymphoma. Int J Hematol 2015;102(4):413-419.

22. Feugier P, Van Hoof A, Sebban C, Solal-Celigny P, Bouabdallah R, Fermé C, et al. Long-term results of the R-CHOP study in the treatment of elderly patients with diffuse large B-cell lymphoma: a study by the Groupe d'Etude des Lymphomes de l'Adulte. J Clin Oncol 2005;23(18):4117-4126. 